УДК 338.012

\title{
С. С. Хворостянников
}

Национальный Исследовательский Ядерный Университет «МИФИ» Обнинский Институт Атомной Энергетики, Обнинск, e-mail: sergey_hvorostyannikov@mail.ru

\section{КОНКУРЕНТОСПОСОБНОСТЬ АТОМНОЙ ПРОМЫШЛЕННОСТИ, КАК ЭКОНОМИЧЕСКАЯ КАТЕГОРИЯ}

Ключевые слова: конкурентоспособность, атомная промышленность, экономическая категория, национальная экономика.

В статье рассматриваются теоретико-методологические аспекты конкурентоспособности атомной промышленности, как экономической категории. Развитие конкурентоспособности атомной промышленности является одним из приоритетных направлений развития национальной экономики Российской Федерации. В целях выявления способов определения конкурентоспособности атомной промышленности на мировом рынке атомной энергетики, проведён анализ теоретических аспектов категории «конкурентоспособность отрасли». Актуальность данного вопроса обусловлена тем, что развитие национальной экономики напрямую связано с конкурентоспособностью её основных отраслей, одной из которых является атомная промышленность. Ряд отечественных и зарубежных авторов активно занимаются изучением конкурентоспособности, при этом предлагаемые экономистами модели в значительной мере отличаются друг от друга. Целями данного исследования явились сравнительный анализ отечественных и зарубежных моделей конкурентоспособности, классификация факторов категории «конкурентоспособность атомной промышленности». В статье рассмотрены различные факторы конкурентоспособности отрасли. В ходе работы определены основные показатели и факторы, влияющие на категорию «конкурентоспособность атомной промышленности», проведена классификация конкурентоспособности по группам. Результатом исследования является: классификация факторов конкурентоспособности атомной промышленности по показателям. Также в статье предлагается авторское определение категории «конкурентоспособность атомной промышленности».

\section{S. S. Khvorostyannikov}

Obninsk Institute for Nuclear Power Engineering, Obninsk, e-mail: sergey_hvorostyannikov@mail.ru

\section{METHODOLOGICAL ACCENTS OF COMPETITIVENESS OF ATOMIC ENERGY}

Keywords: competitiveness, nuclear industry, economic category, national economy.

The article discusses the theoretical and methodological aspects of the competitiveness of the nuclear industry as an economic category. The development of the competitiveness of the nuclear industry is one of the priority areas for the development of the national economy of the Russian Federation. In order to identify ways to determine the competitiveness of the nuclear industry in the global nuclear energy market, an analysis of the theoretical aspects of the category «industry competitiveness» is carried out. The relevance of this issue is due to the fact that the development of the national economy is directly related to the competitiveness of its main industries, one of which is the nuclear industry. A number of domestic and foreign authors are actively engaged in the study of competitiveness, while the models proposed by economists are significantly different from each other. The objectives of this study were a comparative analysis of domestic and foreign models of competitiveness, classification of factors of the category "«ompetitiveness of the atomic industry». The article discusses various factors of industry competitiveness. In the course of the work, the main indicators and factors influencing the category of «competitiveness of the nuclear industry» were determined, and classification of competitiveness by groups was carried out. The result of the study is: classification of the competitiveness factors of the nuclear industry by indicators. The article also offers an author's definition of the category «competitiveness of the nuclear industry».

\section{Введение}

В современном мире множество факторов оказывают влияние на международные экономические отношения. Одним из таких факторов является глобализация конкуренции. Основной целью экономики Российской Федерации является её рост и укрепление на мировом рынке. Для достижения этой цели необходимо активное участие на мировом рынке и наличие высококонкурентных отраслей национальной экономики. Одной из таких отраслей в Российской Федерации является атомная промышленность. Основой конкурентоспособности атомной промышленности является 
процесс модернизации её производства и улучшение качества товаров. В связи с этим необходимо рассмотрение конкурентоспособности атомной промышленности, как экономической категории.

\section{Цель исследования}

Разработка методологических основ категории «конкурентоспособность атомной промышленности», включая определение данного понятия и выявление его факторов.

\section{Материал и методы исследования}

В рамках изучения данной проблемы использовался формально-логический метод исследования. Информационной базой исследования послужили труды отечественных и зарубежных авторов по исследованию категории «конкурентоспособность».

\section{Результаты исследования}

Для разработки теоретических методов и подходов к формированию категории «конкурентоспособность атомной промышленности», необходимо, прежде всего, рассмотреть категорию «конкурентоспособность».

При рассмотрении категории «конкурентоспособность» можно выделить следующие характеристики данного понятия, как для отдельных субъектов и отраслей, так и для всей экономики в целом:

1. экономические;

2. научно-технические;

3. производственные;

4. организационно-управленческие;

5. маркетинговые.

Приведённые характеристики подтверждают важность категории «конку- рентоспособность» для национальной экономики. Принцип конкурентоспособности также является основным для рыночной экономики. Оценка конкурентоспособности отрасли и её анализ производятся с помощью различных научных теорий и их положений, однако практически все они достаточно точно показывают структуру рынка.

При детальном рассмотрении становится, очевидно, что конкурентоспособность представляет собой социальноэкономическую категорию достижения максимально возможным преимуществ на рынке всеми законными способами. При рассмотрении категории «конкурентоспособность» стоит чётко определять уровень её рассмотрения, поскольку в зависимости от уровня методы анализа и оценки будут различаться. По этой причине все экономические объёкты, к которым можно применить понятие конкурентоспособности делятся на четыре группы:

1. товары;

2. предприятия, производящие товары и услуги;

3. отрасли;

4. регионы, включая в себя районы, области, страны и целые группы.

В табл. 1 представлена классификация конкурентоспособности по группам.

Принадлежность объекта к той или иной группе позволяет выбрать наиболее подходящую стратегию конкурентной борьбы на локальном или глобальном рынке. Исходя из этого, можно сказать, что существует четыре вида конкурентоспособности:

1. конкурентоспособность товара;

2. конкурентоспособность предприятия;

Таблица 1

Классификация конкурентоспособности по группам

\begin{tabular}{|c|c|l|}
\hline $\begin{array}{c}\text { Объект конкуренто- } \\
\text { способности }\end{array}$ & Уровень & \multicolumn{1}{|c|}{ Проявление конкурентоспособности } \\
\cline { 1 - 2 } Товар & Микроуровень & $\begin{array}{l}\text { Устойчивая конкурентоспособность на внутреннем или внеш- } \\
\text { нем рынках }\end{array}$ \\
\cline { 3 - 3 } Предприятие & $\begin{array}{l}\text { Способность находить конкурентные преимущества для устой- } \\
\text { чивого развития }\end{array}$ \\
\hline Отрасль & Мезоуровень & $\begin{array}{l}\text { Эффективное использование государственных программ для } \\
\text { повышения конкурентоспособности отрасли }\end{array}$ \\
\hline Регион & Макроуровень & $\begin{array}{l}\text { Совокупная отраслевая конкурентоспособность с учётом эф- } \\
\text { фективных макроэкономических показателей }\end{array}$ \\
\hline
\end{tabular}


3. конкурентоспособность отрасли;

4. национальная конкурентоспособность.

Основоположником современного понятия категории «конкурентоспособность» по праву считается М. Портер. Его определение категории «конкурентоспособность» строится на принципе: только конкурентно способные предприятия отрасли могут сформировать общеотраслевую конкурентоспособность, обеспечивающую конкурентоспособность национальной экономики на мировом рынке [1].

В своих работах по изучению конкуренции и конкурентоспособности, Портер предлагает модель конкурентного ромба [1]. В этой модели он выделяет следующие факторы:

1. Факторные условия: наличие людских и природных ресурсов; высокий интеллектуальный и информационный потенциал; наличие инвестиционного капитала и соответствующей инфраструктуры.

2. Условия внутреннего спроса: соответствие выпускаемой продукции, требованиям рынка; изменение динамики производства в соответствии с изменением объёмов спроса; соответствие тенденциям спроса на внутреннем и внешнем рынках.
3. Смежные и обслуживающие отрасли: присутствие на внутреннем рынке поставщиков и смежных отраслей производства, конкурентоспособных на мировом рынке.

4. Внутриотраслевая конкуренция: наличие фирм и компаний с эффективными стратегиями развития; конкурентоспособный менеджмент компаний; различные формы организации производства.

Схематично данная модель может быть представлена следующим образом (рис. 1).

По мнению Портера на внутриотраслевую конкуренцию оказывают воздействие пять факторов. По уровню их воздействия можно определить интенсивность конкуренции. Данные факторы соответствуют пяти силам конкуренции, определённых Портером [2].

1. Возможность появления внутри отрасли новых конкурентов.

2. Возможность появления товаров субститутов.

3. Способность существующих в отрасли компаний конкурировать между собой.

4. Возможность поставщиков расходных материалов участвовать в торгах.

5. Способность производителей и покупателей договариваться о цене.

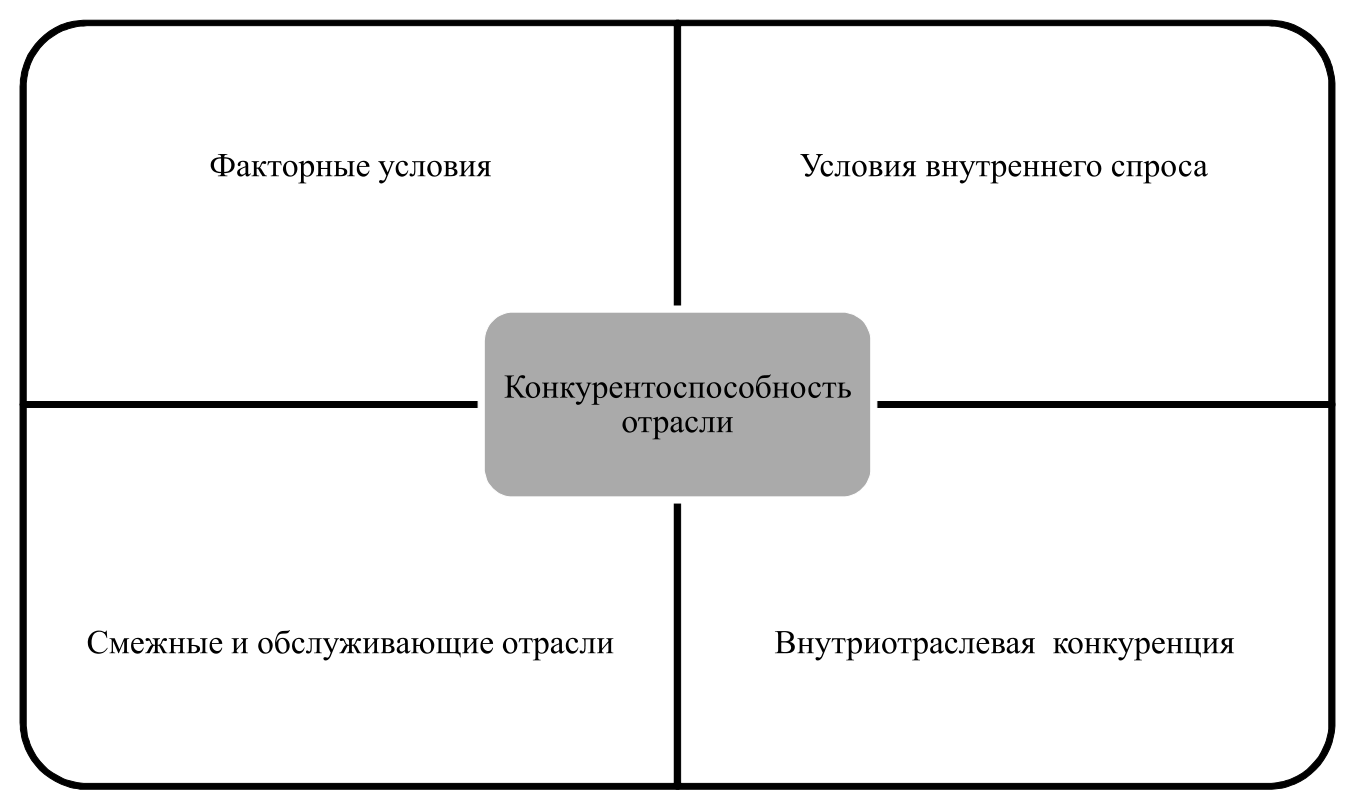

Рис. 1. Модель конкурентного ромба Портера 


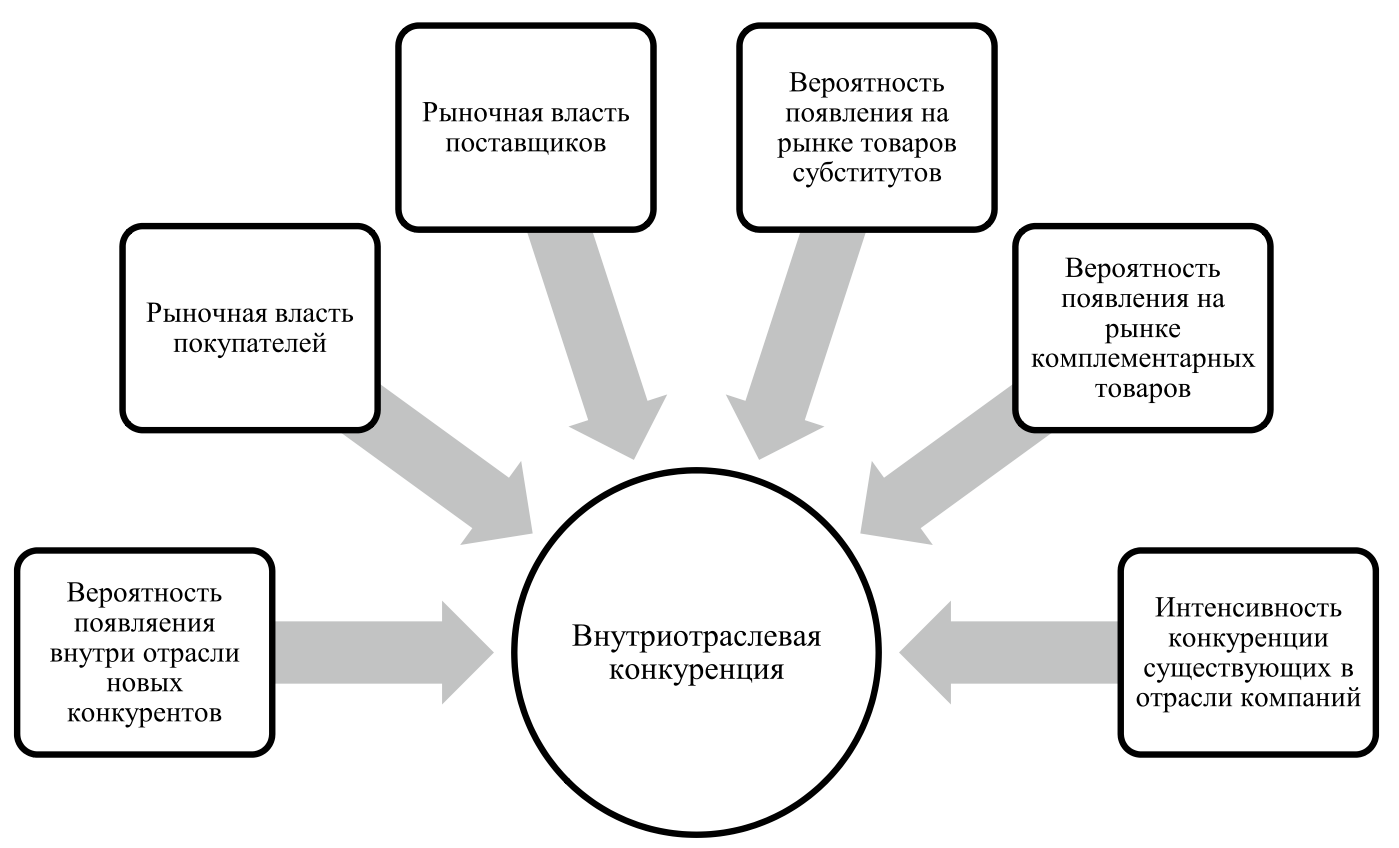

Рис. 2. Модель «шести сил»

Стоит заметить, что данная модель может быть дополнена шестой силой возможность появления в отрасли комплементарных товаров. С учётом шестой силы конкуренции, модель Портера может быть представлена следующим образом (рис. 2).

Рассмотрение влияния шестой сила, вероятности появления комплиментарных товаров, является особо актуальным для атомной промышленности. Поскольку успех реализации большинства крупных атомных проектов связан со смежными областями производства.

Однако, также стоит заметить, что в современных работах учёных-экономистов присутствуют различные определения категории «конкурентоспособность отрасли». Исходя из этого, можно смело сказать, что нет единого понимания данной категории.

Если обратиться к определению категории «конкурентоспособность отрасли» даваемое Организацией экономического сотрудничества и развития, то заметим следующее: конкурентоспособность отрасли рассматривается с точки зрения генерирования высокого уровня доходов для национальной экономики на мировом открытом рынке [3].

1. Уровень динамики ориентации национального производства.
2. Уровень импорта готовой продукции на внутреннем рынке и его динамика.

3. Уровень развития технологий и объём инвестиционного капитала.

4. Уровень конкурентоспособности национальных компаний в сравнении с уровнем мировых лидеров в различных отраслях.

5. Уровень сырьевой базы, кооперационных связи, историческая «привязанность» потребителей к производителям;

6. Отсутствие теневого сектора экономики, уровень правовой базы.

Данная факторная модель позволяет оценивать конкурентоспособность отрасли, не только с аналогичными отраслями в мировой экономике, но и с другими отрасли внутри национальной экономики. Позволяет оценить инвестиционную привлекательность и её перспективе на мировом рынке.

Способность приспосабливаться к быстро меняющимся условиям международного рынка в условиях экономической интернационализации является ключевым фактором международной конкурентоспособности отрасли. Стоит отметить, что быстроменяющиеся условия международного рынка включают в себя различные угрозы и возможности повышения уровня конкурентоспособности, которые возникают при транс- 
граничном сотрудничестве в отрасли различных промышленных предприятий. Достижение целей международной экономической деятельности напрямую зависит от возникающих возможностей и угроз на релевантном рынке, которые влияют непосредственно на уровень конкурентоспособности предприятий в отрасли.

Отсутствие единого определения категории «конкурентоспособность отрасли» позволяет с различной точностью трактовать те или иные экономические положения, что, несомненно, помогает максимально широко рассмотреть объект исследования. Несмотря на это, данное обстоятельство в большой степени усложняет процесс оценки и определения факторов конкурентоспособности.

Исходя из вышесказанного, можно сделать вывод, что категория конкурентоспособность отрасли является частью многоуровневой категории «конкурентоспособность». Характер отраслевой конкуренции на внутреннем и внешнем рынках находит отражением именно в данной категории. Важными факторами конкурентоспособности отрасли является благоприятный инвестиционный климат, открытая экономика и наличие на рынке большого числа конкурирующих субъектов.

Характер отраслевой конкуренции на внутреннем и внешнем рынках нахо- дит отражением именно в данной категории. Важными факторами конкурентоспособности отрасли является благоприятный инвестиционный климат, открытая экономика и наличие на рынке большого числа конкурирующих субъектов.

Конкурентоспособность национальной экономики во многом формируется конкурентоспособностью отраслей экономики, а также конкурентоспособностью её субъектов, при наличии ряда факторов, что обуславливает актуальность изучения категория «конкурентоспособность атомной промышленности». Исходя из рассмотренных выше моделей, можно сделать вывод, что конкурентоспособность атомной энергетики предстаялет собой совокупность конкурентных позиции данной отрасли на различных уровнях рынка с различными видами конкуренции.

Важность данного исследования обусловлена необходимостью интеграции экономики Российской Федерации в мировую экономику, где преобладают конкурентные связи. Изучение категории «конкурентоспособность атомной промышленности» вызвано тем, что данная отрасль является одной из основных отраслей национальной экономики Российской Федерации. По результатам анализа построена таблица классификации факторов конкурентоспособности отрасли (табл. 2).

Таблица 2

Классификация факторов конкурентоспособности атомной промышленности

\begin{tabular}{|c|c|c|c|}
\hline № & Показатель & Фактор & Параметр \\
\hline \multirow[t]{5}{*}{1} & \multirow[t]{5}{*}{ Важность } & Для субъектов отрасли & $\begin{array}{l}\text { Социально-экономический показатель стратегического } \\
\text { развития предприятия или иного субъекта отрасли }\end{array}$ \\
\hline & & Для государства & $\begin{array}{l}\text { Социально-экономический показатель стратегического } \\
\text { развития государства с учётом геополитического состоя- } \\
\text { ния страны }\end{array}$ \\
\hline & & $\begin{array}{c}\text { Для международных } \\
\text { образований }\end{array}$ & $\begin{array}{l}\text { Социально-экономический показатель приоритетов от- } \\
\text { расли, с учётом уровня международных отношений }\end{array}$ \\
\hline & & Для инвесторов & Различные экономические показатели \\
\hline & & Для потребителей & $\begin{array}{l}\text { Различные социальные показатели (стоимость, надёж- } \\
\text { ность, доступность и т. д.) }\end{array}$ \\
\hline \multirow[t]{2}{*}{2} & \multirow[t]{2}{*}{ Измеряемость } & Измеряемый & Количественный показатель (измеряется объективно) \\
\hline & & Неизмеряемый & Качественный показатель (измеряется субъективно) \\
\hline \multirow[t]{2}{*}{3} & \multirow[t]{2}{*}{$\begin{array}{l}\text { Результат взаи- } \\
\text { модействия }\end{array}$} & Стимулирующий & $\begin{array}{l}\text { Показатель способности конкурентоспособности отрасли } \\
\text { к росту на внешнем и внутреннем рынках }\end{array}$ \\
\hline & & Депрессивный & $\begin{array}{l}\text { Показатель снижения конкурентоспособности отрасли на } \\
\text { внешнем и внутреннем рынках }\end{array}$ \\
\hline
\end{tabular}




\begin{tabular}{|c|c|c|c|}
\hline \multicolumn{4}{|r|}{ Окончание табл. 2} \\
\hline № & Показатель & Фактор & Параметр \\
\hline \multirow[t]{2}{*}{4} & \multirow[t]{2}{*}{$\begin{array}{c}\text { Возможность } \\
\text { переноса }\end{array}$} & Полный перенос & $\begin{array}{l}\text { Показатель переноса субъекта отрасли или его копируе- } \\
\text { мости (возможен полный перенос или полное копирова- } \\
\text { ние структуры субъекта отрасли) }\end{array}$ \\
\hline & & Частичный перенос & $\begin{array}{l}\text { Показатель частичного переноса субъекта отрасли или его } \\
\text { частичная копируемость (возможен лишь частичный пере- } \\
\text { нос или частичное копирование из-за ряда ограничений) }\end{array}$ \\
\hline \multirow[t]{2}{*}{5} & \multirow[t]{2}{*}{ Управляемость } & Контролируемый & $\begin{array}{l}\text { Возможность государства или субъектов отрасли влиять } \\
\text { на отрасль. }\end{array}$ \\
\hline & & Неконтролируемый & $\begin{array}{l}\text { Возможность влияния государства или субъектов на от- } \\
\text { расль полностью исключена. }\end{array}$ \\
\hline \multirow[t]{2}{*}{6} & \multirow{2}{*}{$\begin{array}{c}\text { Среда функцио- } \\
\text { нирования }\end{array}$} & Внутренний & Формируется внутри отрасли её субъектами. \\
\hline & & Внешний & Формируется за пределами отрасли. \\
\hline \multirow[t]{4}{*}{7} & \multirow[t]{4}{*}{$\begin{array}{l}\text { Модель конку- } \\
\text { рентного ромба } \\
\text { Портера }\end{array}$} & Ресурсы & $\begin{array}{l}\text { Человеческие и природные ресурсы, инвестиционный } \\
\text { капитал, высокоинтеллектуальный информационный по- } \\
\text { тенциал, развитая инфраструктура }\end{array}$ \\
\hline & & Внутренний спрос & $\begin{array}{l}\text { Показатель уровня спроса, его структуры и соответствия } \\
\text { требованиям потребителей на мировом рынке }\end{array}$ \\
\hline & & $\begin{array}{l}\text { Смежные и обслужи- } \\
\text { вающие отрасли }\end{array}$ & $\begin{array}{l}\text { Наличие или отсутствие смежных и обслуживающих от- } \\
\text { раслей }\end{array}$ \\
\hline & & $\begin{array}{c}\text { Внутриотраслевая } \\
\text { конкуренция }\end{array}$ & $\begin{array}{l}\text { Наличие или отсутствие фирм и компаний с эффективны- } \\
\text { ми стратегиями развития }\end{array}$ \\
\hline
\end{tabular}

\section{Заключение}

По итогам анализа выявлено, что категория «конкурентоспособность атомной промышленности» зависит от семи основных показателей, которые в свою очередь разделяются на факторы.

Также можно дать следующее определение категории «конкурентоспособность атомной промышленности».
Категория «конкурентоспособность атомной промышленности» - это качественная социально-экономическая характеристика, показывающая её позиции на различных уровнях рынка с различными видами конкуренции, определяющая эффективностью деятельности предприятий, входящих в данную отрасль.

\section{Библиографический список}

1. Портер М.Е. Международная конкуренция. Конкурентные преимущества стран. М.: Альпина Пабл., 2016. 947 с.

2. Портер М.Е. Конкурентное преимущество: Как достичь высокого результата и обеспечить его устойчивость. М.: Альпина Пабл., 2016. 715 с.

3. Мищенко Л.Я., Хомутова А.И. Оценка конкурентоспособности национальной экономики: теоретические и методические аспекты // Политематический сетевой электронный научный журнал Кубанского государственного аграрного университета. 2016. № 10(074). С. 710-718.

4. Сальников В.А., Галимов Д.И. Конкурентоспособность отраслей российской промышленности - текущее состояние и перспективы // Проблемы прогнозирования. 2016. № 2. С. 55-84. 